\title{
Klaus Kreimeier
}

\section{Einführung}

Nachdem man die Referate der ersten Sektion unter die Überschrift stellen konnte: »Warum und zu welchem Ende studieren oder lehren wir Medienwissenschaft? «, sind in dieser Sektion an die erste Stelle die Fragen zu rücken: Was treibt eigentlich die Medienwissenschaft, was tut sie in ihrer alltäglichen Praxis? Zweite Frage: Welche Fragen und Forderungen hat die Medienpraxis an die Medienwissenschaft? Und, daran anknüpfend, eine dritte Frage: Kann die heutige Medienwissenschaft der Medienpraxis zu größerer Klarheit verhelfen, kann sie ihr zu einer besseren Praxis verhelfen?

Helmut Korte ist Professor für interdisziplinäre Medienwissenschaft an der Universität Göttingen, dort an der Sozialwissenschaftlichen Fakultät tätig, wo es auch eine Institutionalisierung der Medienwissenschaften gibt in Gestalt des Zentrums für Interdisziplinäre Medienwissenschaft (ZIM), das zuständig ist für den Magisterstudiengang, den Helmut Korte vorstellt. Es wurde vor etwa zwei Jahren in Göttingen gegründet und ist verantwortlich für die Durchführung und den Erfolg dieses Magisterstudiengangs »Medien- und Kommunikationswissenschaft «. Das ZIM versteht sich als Schnittstelle innerhalb der Universität für Lehre und Forschung, leistet also entsprechende Dienstleistungen für die Universität insgesamt als fach- und fakultätsübergreifende Einrichtung. Das Stammpersonal sind die Mitglieder des ehemaligen Instituts für Publizistik und Kommunikationswissenschaft in Göttingen, das erweitert wurde durch neu hinzu gekommene Stellen für Film- und Fernsehwissenschaft, Neue Medien und Journalistik. Medien- und Kommunikationswissenschaft streben hier nach einer Synthese. Helmut Korte möchte einige idealtypische Fachkonzeptionen skizzieren, die er zumindest in Ansätzen in dem neu eingerichteten Studiengang »Medien- und Kommunikationswissenschaft « in Göttingen realisiert sieht und dort erproben kann. In seinem Papier geht es Helmut Korte im Wesentlichen um vier Punkte: Erstens um Interdisziplinarität, verstanden als wechselseitige Interdisziplinarität zwischen den beteiligten Fächern und Arbeitsbereichen, also um ein Geben und Nehmen aufgrund der Komplexität des Gegenstandes, um die Integration von Erkenntnissen, Theoriebildungen und Methoden aus zahlreichen Fächern um das Fach Medienwissenschaft, darum auch, die gebündelte Medienkompetenz in diesem Zentrum für Interdisziplinäre Medienwissenschaft auch anderen Fächern zugute kommen zu lassen. Zweiter Punkt: Helmut Korte ist wichtig, dass der Graben oder die Kluft zwischen Medien- und Kommunikationswissenschaft überwunden wird, dass es zu einer besseren Kooperation kommt zwischen einer primär philologisch ausgerichteten Film- und Fernsehwissen- 
schaft und einer stärker sozialwissenschaftlich orientierten Publizistik und Kommunikationswissenschaft. Diese Union, diese Re-Union zwischen zwei unterschiedlichen Strömungen, die ja einen ähnlichen historischen Ansatz haben, dann aber unterschiedliche Entwicklungen durchlaufen haben, ist in Göttingen offenbar auf dem besten Wege. Dritter Punkt: Mediale Breite. Dabei es geht um eine Vielfalt von Medien, klassische technische Medien und die so genannten Neuen Medien, darum also, dieses breite Spektrum sowohl im Curriculum wie in der Forschung wahrzunehmen und auch zu realisieren. Vierter Punkt: Es geht um fach- und berufspraktische Qualifikationen, also um die Verbindung zwischen wissenschaftlicher Orientierung und Praxisorientierung im Curriculum und in den Forschungsansätzen. Helmut Korte stellt dann in aller Kürze Film- und Fernsehwissenschaft dar als zentrale medienwissenschaftliche Fachgegenstände mit dem Kern der Medienanalyse, wie sie sich aus den Kulturwissenschaften entwickelt haben, auch aus den Philologien - Medienanalyse verstanden als Einheit von Produktionsanalyse, Produktanalyse und Rezeptionsanalyse. Er hebt hervor, dass gerade die Film- und Fernsehwissenschaft als Teilgebiet der Medienwissenschaft prinzipiell durch Interdisziplinarität gekennzeichnet ist und schon deshalb eine inhaltliche und methodische Offenheit zu anderen Methodologien haben muss.

Joachim Paech ist Professor für Medienwissenschaft an der Universität Konstanz, dort für Theorie und Geschichte des Films verantwortlich, und hat ein besonderes Forschungsinteresse im Bereich der Intermedialität zwischen Film, Literatur und den klassischen Künsten. Sein Papier trägt die Überschrift: »Für eine Filmgeschichte der Medienwissenschaft«. Joachim Paech geht zunächst historisch vor und teilt die Filmgeschichte in vier Epochen ein. Er bezieht sich dabei weniger auf die Entwicklung des Films selbst oder der Filmtechnik, sondern in erster Linie auf die Diskurse, die in diesen jeweiligen Perioden oder Epochen vom Film und von der Entwicklung des Films ausgelöst wurden. Die erste Epoche steht unter der Überschrift »Der Film als Reproduktionstechnik etwa von 1895 bis 1910 «. Verstanden darunter ist der Diskurs über das Kino, der die Kinematographie als technisch-apparative Erweiterung der Photographie zum Bewegungsbild versteht, also etwa die ersten 15 Jahre der Kinematographie. Die zweite Epoche »Der Film als Kunst und Werk « ist die weitaus größte Zeitspanne, etwa vom Beginn der 1910er Jahre bis 1960. In diesen Jahrzehnten liefen mehrere Diskurse zusammen. Der Film wird verstanden als Paradigma der Paradoxien der Moderne. Er sei einerseits Wahrnehmung, andererseits auch Darstellung von Modernität, und es kommen auch traditionelle Diskurse in diesem neuen Mediendiskurs zusammen. Und zwar stammen sie aus den Traditionen des epischen Erzählens im 19. Jahrhundert einerseits und dem Theater als einem traditionellen Darstellungsdispositiv andererseits, das gleichermaßen aus dem 19. Jahrhundert überliefert wird, also eine Fortsetzung bürgerlicher ästhetischer und kultureller Traditionen, nun mit technischen Mitteln und aufgehend in einem neuen, komplexen, medialen Diskurs. Joachim Paech verweist in diesem Zusammen- 
hang auf einige wichtige Theoretiker dieser Periode, natürlich Walter Benjamin, der den Film als Kunstwerk und als Medium zugleich verstanden hat, dann auf die großen Phänomenologen wie Erwin Panofsky, André Bazin oder Jean Mitry, die den Film als Vermittler zwischen der Realität als Rohstoff und dessen symbolischer Verarbeitung mit ontologischen Ansätzen beschrieben haben. Joachim Paech bezieht sich auch kurz auf die Autorentheorie des Films, die ja stets den Werkcharakter hervorgehoben hat, die Einmaligkeit des Werkes und die Autonomie des Werkes gegenüber dem Medium und seiner Technik betonte, auch deswegen, weil die Autoren, die sich damals in den sechziger, siebziger Jahren für ihre Autorentheorie eingesetzt haben, mit einem neuen Massenmedium konfrontiert waren, nämlich dem Fernsehen. Die dritte, etwas kürzere Phase beschreibt Joachim Paech als die intertextuelle Phase des Films. Sie sei anzusetzen für den Übergang von der Kunsttheorie des Films zur Texttheorie, Text im nachdrücklichen Sinn verstanden als zunächst basierend auf der Texttheorie der Literatur, dann aber erweitert um strukturalistische, semiotische und linguistische Aspekte zu einem erweiterten Textbegriff, der sich seine eigene Wissenschaftstradition im Zusammenhang mit dem Film, der zunehmend als »langue«, als Sprache verstanden wird, schafft. Die vierte Phase, mit der nach Paech die Filmgeschichte und auch die Diskursgeschichte des Films, soweit sie sich wirklich auf die Kinematographie konzentriert, zum Abschluss kommt, sei die intermediale Phase. Der intermediale Film seit Beginn der achtziger Jahre etwa, knüpft in bezeichnender Weise an die modernen Anfänge der Jahrhundertwende medienreflexiv an, das cinema of attraction der frühen Jahre wird wieder entdeckt, zu Recht, und in den modernen Diskurs einbezogen. Die Mediendefinition des Films stellt nach Paech über seine kulturelle Codierung hinaus den Film in den Kontext der Medientechnologien von heute und ist damit nur noch ein bestimmtes technisch-apparatives Medium unter anderen. Der Film ist nur noch eine mediale Form, die unterschiedlich, und zwar analog wie heute auch digital, realisiert werden kann. Diese neue Intermedialität des Films ordnet ihn ein in eine zweite, reflexive Moderne und löst damit die Singularität des Mediums, die jahrzehntelang behauptet wurde, im Multimedialen auf. Das heißt, Medienwissenschaft - und das ist die Pointe dieses Papier - kann es erst geben, seit die Differenzierung der ersten Moderne in die verschiedenen Institutionen der Künste und andere soziale Institutionen und deren Rückwirkung auf den Wissenschaftsdiskurs zunächst in globalen Zeichensystemen und dann in medialen Konfigurationen ihrer Ermöglichung aufgehoben worden sind. Medienwissenschaftlich ist der Film nunmehr eine Form medialer Realisierung im multimedialen Kontext. Joachim Paechs Referat erzählt uns in aller Kürze die Geschichte der Ermöglichung von Medienwissenschaft als Filmgeschichte.

Knut Hickethier ist Professor für Medienwissenschaft an der Universität Hamburg. Seine Schwerpunkte sind dort Filmanalyse und die Geschichte des deutschen Fernsehens. Ich erlaube mir zur Vorstellung des Papiers eine kleine 
Montage insofern, als ich mich erst dem Mittelteil widme, der nämlich sehr gut anschließt an die Papiere von Helmut Korte und Joachim Paech. Dann werde ich die Präambel, die Knut Hickethier seinem Papier vorangestellt hat, referieren und den angehängten Schluss in einem zweiten Schritt zusammenfassen. Knut Hickethier schreibt: »Film- und Fernsehwissenschaft sind nach meinem Verständnis und wie sie an der Universität Hamburg betrieben werden, nicht separate Wissenschaften, sondern Teil des Faches Medienwissenschaft bzw. Medienkultur.«Dies ist ein fast wörtlicher Anschluss an die Position von Helmut Korte. Zweiter Punkt, zweite These: »Die wissenschaftliche Beschäftigung mit Film und Fernsehen ist in Hamburg text- und kulturwissenschaftlich ausgerichtet, wobei der Textbegriff auch für audiovisuelle Produkte gilt. «Dies ist ein deutlicher Anschluss an die Thesen von Joachim Paech. Im Kulturbegriff ist einerseits das Konzept Text als Kultur und Kultur als Text integriert. Andererseits wird Kultur als kulturelle Praxis verstanden. Damit werden der Begriff der medialen Beobachtung wie der der Kommunikation gefasst, die wiederum unterschiedliche Formen der Produktion und Rezeption einschließen. Die kulturwissenschaftliche Ausrichtung der Beschäftigung mit Film und Fernsehen stellt die medialen Produkte als mediale Texte in den Vordergrund. Dritter Punkt: Die Medienwissenschaft befasst sich an zentraler Stelle mit den technisch-apparativen Medien, die drei kulturell dominante Funktionen miteinander verbinden, und zwar die Formen der medialen Beobachtung, der medialen Speicherung und Überarbeitung sowie die mediale Vermittlung. Vierte These: Die Beschäftigung mit Film und Fernsehen sollte davon ausgehen, dass einerseits der mediale Rahmen mit den Aspekten der Medialität, also der jeweils unterschiedlichen Medialität des Kinematographischen und des Televisuellen, zu bestimmen sei. Andererseits seien innerhalb dieses Rahmens auch die Bedeutungen, die Inhalte, die Themen, Master Narratives, Geschichten und Genres von zentraler Bedeutung. Eine fünfte These geht kurz auf die Arbeitsfelder Medienanalyse, Medientheorie und Mediengeschichte ein als kulturwissenschaftliche Kernbereiche jeglicher Medienwissenschaft. Die sechste These ist sehr markant formuliert: »Medienwissenschaft ist zwangsläufig schon von ihrem Gegenstandsbereich her international und nicht national ausgerichtet.« Und betont dann noch einmal mit Nachdruck: »Dies bedeutet auch ein Wissen um die eigenen medienwissenschaftlichen Traditionen und Kenntnis der eigenen Fachpositionen; wir sind wissenschaftlich keine Kultur zum Beispiel der amerikanischen Mediendepartments. «Diese Thesen zur Film- und Fernsehwissenschaft rahmt Hickethier ein in eine hochschulpolitische Positionsbestimmung, die ihrerseits sehr pointiert ist, eine These, die an Klarheit nichts zu wünschen übrig lässt. Knut Hickethier spricht von »Abgrenzung «, zweimal und in aller Entschiedenheit, auch, um vom eigenen Standpunkt aus wiederum mit der Kommunikationswissenschaft und mit anderen Kultur- und Textwissenschaften kooperieren zu können. Nicht Abgrenzung also, um eine eigene Hegemonie abzustecken, ist gemeint, sondern um die Zusammenarbeit und die Koope- 
ration, die heute defizitär ist, zu verbessern oder überhaupt erst in die Wege zu leiten.

Ich müsste jetzt sagen »Szenenwechsel« oder »Themenwechsel«, denn wir kommen jetzt zu einem zentralen Thema, nämlich der Medienpraxis und den Fragen, die die Medienpraxis an die Wissenschaft hat. Dieser Positionsbestimmung aus der Praxis liegt ein Papier von Ulrich Demmer vor, Mitarbeiter des WDR, dort verantwortlich in der Abteilung Koordination Informationstechnologie (IT) und seit circa drei Jahren auch damit befasst, die Verantwortung zu dezentralisieren. IT wird im WDR definiert als >computergestützte, vernetzte Arbeitsplätze <. Herr Demmer beschreibt das, was darunter zu verstehen ist, als Dezentralisierung. Ein wichtiger Aspekt dieser Dezentralisierung im WDR ist, so Ulrich Demmer, die Beteiligung des internen Kunden, also der einzelnen Redaktionen, der Abteilungen, derjenigen, die die $>$ Contents< herstellen, an der Technik. Zwischen der dezentralen Organisation und der Geschäftsleitung wurde eine Art Tower geschaffen, auch dies ist eine Information, die interessant ist. Denn das ist eine Sprache, die sich inzwischen in öffentlich-rechtlichen Anstalten durchzusetzen beginnt angesichts komplexer gewordener interner Strukturen. Ein Tower, der den internen IT-Einsatz steuern und weiterführende IT-Strategien gemeinsam mit den Beteiligten entwickeln soll - das ist die Abteilung Koordination IT. Ulrich Demmers Tätigkeit bedeutet, auf eine Formel gebracht, an einer Schnittstelle zu arbeiten zwischen Programm und Technik und dafür zu sorgen, dass die richtigen Entscheidungen im Spannungsfeld zwischen Kunde und Dienstleister getroffen werden. Kunde wird immer verstanden als interner Kunde innerhalb des komplexen Gebildes WDR, die Wünsche zum Beispiel, die eine Abteilung oder eine Redaktion an die Technik hat, um in diesem Spannungsfeld zu operieren und zu richtigen Entscheidungen zu kommen. Ulrich Demmers Positionspapier fasse ich in aller Kürze zusammen. Er sagt, Medienwissenschaft solle keine Rahmenwissenschaft sein, die gewissermaßen nur wiederum ihren hegemonialen Bereich absteckt. Sie hat Transferleistungen zur Verfügung zu stellen und mit ihrem eigenen gewachsenen heterogenen Schatz an Theorien und Methoden mit anderen Disziplinen zu kommunizieren, ein Gedanke, der in den anderen Papieren ja auch schon in aller Deutlichkeit ausgedrückt wird. Demmer geht von der Erkenntnis aus, dass die Medien in komplexen Prozessen beeinflussen und auch beeinflusst werden. Sie seien Subjekt und Objekte und als solche ein maßgeblicher Teil unseres Alltagsgeschehens. Herr Demmer geht dann in vier Punkten, in die er sein Papier untergliedert hat, kurz auf den kulturellen Aspekt ein. Überschrift ist hier »Kulturelle Nachhaltigkeit« vor dem Hintergrund der gegenwärtigen Globalisierungsprozesse. Er sagt, die Ökonomie sei auf dem Vormarsch, und die Globalisierung stehe ihrerseits unter dem Druck der Ökonomie. Gerade deswegen müssten sich die öffentlich-rechtlichen Anstalten auch auf ihren kulturellen Auftrag besinnen. Er führt hier den Begriff der kulturellen Nachhaltigkeit ein, den er am Exempel des europäischen Films verdeutlicht. Ulrich 
Demmer fügt allerdings hinzu, eine rein kulturwissenschaftliche Betrachtung dieses Problems würde diesem Thema ebenso wenig gerecht wie eine rein wirtschafts- oder politikwissenschaftliche Betrachtung. Hier müsse vieles zusammenkommen, um das Phänomen erst einmal zu beschreiben. Solche Verträge wie das Dienstleistungsabkommen GAZ oder die Maßnahmen der Welthandelsorganisationen überhaupt wahrzunehmen und gleichzeitig über die Konsequenzen und über mögliche Gegenstrategien nachzudenken, geschieht in der Medienwissenschaft viel zu peripher. Ein weiterer Gesichtspunkt ist die Öffentlichkeit. Öffentlichkeit beschreibt Ulrich Demmer als eine zunehmende Vielfalt unterschiedlicher Lebensstile in der Gesellschaft, und das ist seine Vermutung - er versieht sie mit einem kleinen Fragezeichen. Offenbar wird das Radio, aber eben auch das Fernsehen in den Angeboten emotionaler. Dann ein Widerspruch, eine verzwickte Ambivalenz, die ja auch vielleicht eine Dialektik sein könnte, wo beide Seiten sich gegenseitig befruchten, aber die doch in der Regel als starrer Gegensatz wahrgenommen wird: nämlich einerseits die immense kulturelle und politische demokratiekonstitutive Bedeutung insbesondere der Massenmedien in unseren hochkomplexen Gesellschaften und andererseits die Rasanz, mit der die Ökonomisierung und Globalisierung eben auch der Strukturen dieser Medien sich bemächtigen, diese Medien verändern. Die Medien müssen ihrerseits im Grunde wie Warenhäuser reagieren, das heißt ständig Programme vernichten, um neuen Bedarf zu schaffen, Mangel wiederherzustellen, Mangel zu organisieren. Es kann nicht ohne cross-mediale Bezüge gehen, gerade wenn man in der Fernsehpraxis arbeitet. Am Ende formuliert Ulrich Demmer Forderungen und Wünsche an die Medienwissenschaft, die im Zentrum auch unseres Gespräches stehen sollten.

Helmut Schanze ist Professor für Literatur- und Medienwissenschaft an der Universität Siegen. Er war lange Jahre Sprecher des DFG-Sonderforschungsbereichs $240 »$ Bildschirmmedien«. Er hat ein außerordentlich komplexes und umfassendes Papier vorgelegt, in dem er ein Modell der integralen Vernetzung der Medienwissenschaften, sowohl im historischen Sinne der Entwicklung der Medienwissenschaft, wie wir sie heute haben, als auch in der Breite mit den verwandten Disziplinen, darstellt. Ein zentraler Absatz beleuchtet die Frage, ob die gegenwärtigen Medienwissenschaften eigentlich im Stande sind, der Medienpraxis auf die Beine zu helfen und ihr zu höherer Klarheit zu verhelfen. Helmut Schanzes Forderung lautet: »Sie haben die Ausbildungsziele mit zu definieren, nicht in Form von Vorschriften für die Praxis, sondern durch deren Reflexion, durch Theorie im Wortsinn. Sie sind in der Begriffsbildung gefordert und haben über ihre Ergebnisse in der wissenschaftlichen Öffentlichkeit zu berichten, in der stillen Hoffnung, dass diese Ergebnisse, in welcher Form auch immer, von der Medienpraxis zur Kenntnis genommen werden.« 